\title{
Características morfológicas dos dentes de Bradypus variegatus (Schinz, 1825): revisão de literatura
}

\section{Morphological characteristics of the teeth of Bradypus variegatus (Schinz, 1825): literature review}

\author{
Maiara Carolina Lima Santos ${ }^{1}$; Juliana Plácido Guimarães ${ }^{2 *}$; Sandra Peres Ferreira ${ }^{2,3}$
}

\begin{abstract}
RESUMO
A preguiça-comum (Bradypus variegatus) ocorre nas Américas Central e do Sul, estando presente na maior parte dos estados do Brasil. É encontrada em florestas tropicais e subtropicais e possui uma alimentação restrita a folhas. Passa a maior parte do tempo na copa das árvores, indo ao solo apenas para defecar, ou quando não encontra possibilidade de atravessar de uma árvore para outra. Possui hábitos solitários, se associando a outro indivíduo apenas na época de acasalamento e durante os primeiros seis meses do filhote. O status da espécie é de menos preocupante, porém, vem sendo ameaçada por diversas formas de fragmentação de seu habitat, além do aumento do número de acidentes por choques elétricos, apanhe ilegal e atropelamentos. Devido à necessidade de conhecimento técnico sobre particularidades na anatomia dessa espécie, para enfrentar os desafios que as ameaças ocasionam ao resgate e reabilitação, este trabalho teve como objetivo estudar a dentição da espécie Bradypus variegatus, uma vez que a mesma tem sido vítima frequente de traumas que dificultam ou impossibilitam sua recuperação e retorno à natureza.
\end{abstract}

Palavras-chave: Preguiça-comum; Dentição; Medicina da Conservação; Anatomia; Odontologia veterinária.

\section{ABSTRACT}

The Common-Sloth (Bradypus variegatus) occurs in Central and South America, being present in most Brazilian states. It's found in tropical and subtropical forests and has a diet restricted to leaves. This animal spends most of its time in the treetops, going to the ground only to defecate or when it cannot cross from one tree to another. It has solitary habits, associating with another individual only during the mating season and during the first six months of the puppy. The status of the species is of less concern, however, it has been threatened by several forms of fragmentation of its habitat, in addition to the increase in the number of accidents due to electric shocks, illegal beatings and being run over. Due to the need for technical knowledge about particularities in the anatomy of this species, to face the challenges that threats cause to the rescue and rehabilitation, this work aimed to study the dentition of the species Bradypus variegatus, since it has been a frequent victim of traumas that make it difficult or impossible for them to recover and return to nature.

Keywords: Common sloth; Dentition; Conservation Medicine; Anatomy; Veterinary dentistry.

\footnotetext{
${ }^{1}$ Médica Veterinária Autônoma

${ }^{2}$ Centro Universitário São Judas Tadeu

${ }^{3}$ EngenheiroTércio Augusto Garcia Junior Ecological Park

*juvetpg@yahoo.com.br
} 


\section{INTRODUÇÃO}

A espécie Bradypus variegatus (SCHINZ, 1825), conhecida como preguiçacomum, pertence à ordem Pilosa, família Bradypodidae e gênero Bradypus. De acordo com Superina et al. (2008), essa espécie é amplamente distribuída nas Américas Central e do Sul.

Atualmente, o status de conservação desses animais é considerado como menos preocupante pela União Internacional para a Conservação da Natureza (IUCN), tendo, como sua principal ameaça o desmatamento, com fragmentação e perda de habitat, pressionando, dessa forma, os espécimes para perto de estradas e cidades. Além disso a preguiça-comum é caçada e usada para alimentação, fins medicinais ou como animal de estimação e atração turística em diferentes regiões (MORAES-BARROS et al., 2014).

A aproximação da vida urbana a áreas de matas, por conta da expansão imobiliária, é a principal causa de acidentes que levam os animais a óbito, por diversos fatores, como atropelamentos, choques elétricos, caça e captura ilegais, entre outros (MACHADO et al., 2008).

A fim de enfrentar os desafios que essas ameaças ocasionam ao resgate e à recuperação de fauna e tendo em vista as dificuldades e limitações encontradas na reabilitação da preguiça-comum, o objetivo deste trabalho é rever e estudar a dentição de Bradypus variegatus, uma vez que a espécie tem sido vítima frequente de traumas que impossibilitam sua recuperação e retorno à natureza, exigindo que os profissionais da área busquem mais conhecimento para compreender a fisiologia da espécie e, assim, procurar mudar esse quadro.

\section{REVISÃO DE LITERATURA}

\section{Preguiça-comum (Bradypus variegatus)}

A Bradypus variegatus, conhecida também como preguiça-de-óculos, preguiça-de-garganta-marrom ou preguiça-comum, pertence à ordem Pilosa, tal qual os tamanduás (REZENDE et al., 2013).

Possuem o corpo com massa muscular reduzida, cauda curta, membros pélvicos curtos e torácicos longos. Apresentam três garras longas em cada membro, que são estruturas essenciais para sua locomoção, alimentação e defesa (MIRANDA et al., 
2007). Possuem grande mobilidade no pescoço, proporcionada por oito ou nove vértebras cervicais, tendo a capacidade de girar a cabeça em até $270^{\circ}$ (MEDRI et al., 2011).

As preguiças são essencialmente folívoras, sendo bem seletivas e consumindo uma pequena porcentagem de espécies vegetais. A dieta natural de todas elas contém baixos níveis de carboidratos solúveis e é rica em fibra (Plese e Chiarello, 2012). A restrição alimentar torna a adaptação sob cuidados humanos um grande desafio, sendo relatados diversos problemas nutricionais e digestórios, como constipação e timpanismo (DINIZ e OLIVEIRA, 1999).

São animais de baixo metabolismo e hábito arborícola, passando a maior parte do tempo em repouso na copa das árvores, se deslocando pelas extremidades dos galhos, com movimentos muito lentos, descendo ao solo geralmente apenas para defecar a cada sete dias ou quando estão em áreas abertas, sem conexão entre as árvores. Além disso, nadam muito bem (CASSANO, 2006) e podem estar ativas tanto durante o dia quanto à noite (CHIARELLO, 2008)

Outro fator que explica a preferência pela copa das árvores é a capacidade de termorregulação das preguiças, que têm seu comportamento diretamente ligado às alterações climáticas, ficando imóveis nas primeiras horas da manhã e passando a se movimentar, gradativamente, conforme a temperatura se eleva, buscando a parte mais alta das árvores, ficando expostas ao sol, para se aquecer (MIRANDA et al., 2007). Sua temperatura corpórea pode variar de $24^{\circ}$ a $35^{\circ} \mathrm{C}$ (GOFFART, 1971). São particularmente sensíveis a baixas temperaturas, sendo que a prolongada exposição ao frio pode causar hipotermia irreversível (MIRANDA et al., 2007).

Não são de hábitos totalmente solitários, pois se associam a outro individuo do sexo oposto apenas na época de acasalamento. A gestação tem duração de 4 a 7 meses, com média de 19 meses de intervalo, gerando apenas uma cria (BEZERRA et al., 2008; SILVA et al., 2014). O filhote permanece no dorso da mãe até os 6 meses de idade quando se torna apto a viver sozinho em seu habitat (MIRANDA et al., 2007).

A espécie apresenta dimorfismo sexual, podendo-se diferenciar os machos adultos por meio de uma mancha negra com bordas amarelas ou alaranjadas nas costas (SILVA et al., 2014). Esta mancha é denominada especulum (WETZEL, 1985), sendo que, nos filhotes e juvenis, não é possível realizar essa identificação (MORAESBARROS et al., 2006). A diferenciação através do exame da genitália é de difícil 
determinação e a maturação sexual ocorre com até 6 anos, quando atingem o peso e o tamanho de adultos (MIRANDA et al., 2007).

\section{Habitat}

Comparando com outros mamíferos do mesmo porte, a preguiça-comum possui uma área de vida pequena, fato que pode ser explicado pelo seu metabolismo lento e sua dieta folívora, que fazem com que o animal se adapte em áreas reduzidas e com grande disponibilidade de alimento. Podem estar presentes em florestas primárias, secundárias, matas de várzeas, igapós e até mesmo em ambientes degradados, desde que haja oferta de alimento, como por exemplo, áreas com proliferação de embaubeiras (Cecropia sp.), que são comuns em diversos tipos de ambientes (CASSANO, 2006).

Ocorrem da Costa Rica ao Equador, Colômbia, Venezuela, Peru, Bolívia, Argentina e praticamente em todo o Brasil, sendo, portanto, habitantes das regiões de clima tropical e subtropical (SUPERINA et al., 2008). Sua ampla distribuição geográfica se dá devido à sua adaptação aos diferentes ambientes, inclusive os que são produzidos em decorrência do desmatamento. Mas, consequentemente, ocorre a diminuição do número de indivíduos em áreas que sofrem fragmentação, gerando a necessidade de estudos e medidas para a preservação desses animais e de seu habitat (MOREIRA et al., 2014).

As preguiças, por suas características biológicas, ficam particularmente suscetíveis a riscos quando expostas a ambientes desflorestados e com pouca disponibilidade de alimento (CASSANO, 2006).

Possuem hábitos arborícolas e são folívoros (PLESE E CHIARELLO, 2012). Com alto grau de especificidade alimentar, consomem, predominantemente, folhas, principalmente as jovens e as maduras, de diversas espécies de árvores (HAYSSEN, 2010).

Segundo estudos, as plantas mais consumidas são pertencentes às famílias Moraceae (CHIARELLO, 2008), Cecropiaceae e Clethraceae (URBANI e BOSQUE, 2007). As preferências variam de animal para animal e de acordo com a disponibilidade de alimento na região (HAYSSEN, 2010).

\section{Dentição}

Visando a preocupação com o bem-estar de animais silvestres mantidos sob cuidados humanos e em reabilitação, é imprescindível que se tenha o conhecimento 
sobre a espécie, especialmente no que diz respeito às estruturas do aparelho digestório, que determinam e interferem diretamente na dieta e manejo desses animais, destacando as particularidades de sua dentição (ALBUQUERQUE et al., 2016).

O aparelho digestório da preguiça-comum, como em outras espécies, começa na boca, onde se observa uma língua curta e de coloração escura (Figura 1), sem função de apreensão do alimento, mas que é importante para a movimentação do mesmo dentro da boca e que demonstra ser pouco eficiente na manipulação de outros itens que não sejam folhas (GOFFART, 1971).

\section{Macroscopia}

Esses animais apresentam fórmula dentária: I. 0/0, C. 0/0, Pm e M. 5/4 = 18 (LOUREIRO e MONTEIRO, 1993). Os dentes têm tamanhos semelhantes (Figura 2), com exceção do molar anterior que pode ser um pouco menor, ou ausente (MIRANDA, 2007).

Como principais características dos dentes das preguiça-comum, observa-se a ausência de esmalte, o formato cilíndrico, crescimento contínuo, sem raiz propriamente dita e a presença de espaços interdentários, sendo eles morfologicamente especializados para maceração (OLIVEIRA, 2005).

Figura 1 - Cavidade oral de exemplar de B. variegatus, evidenciando dentes e língua.

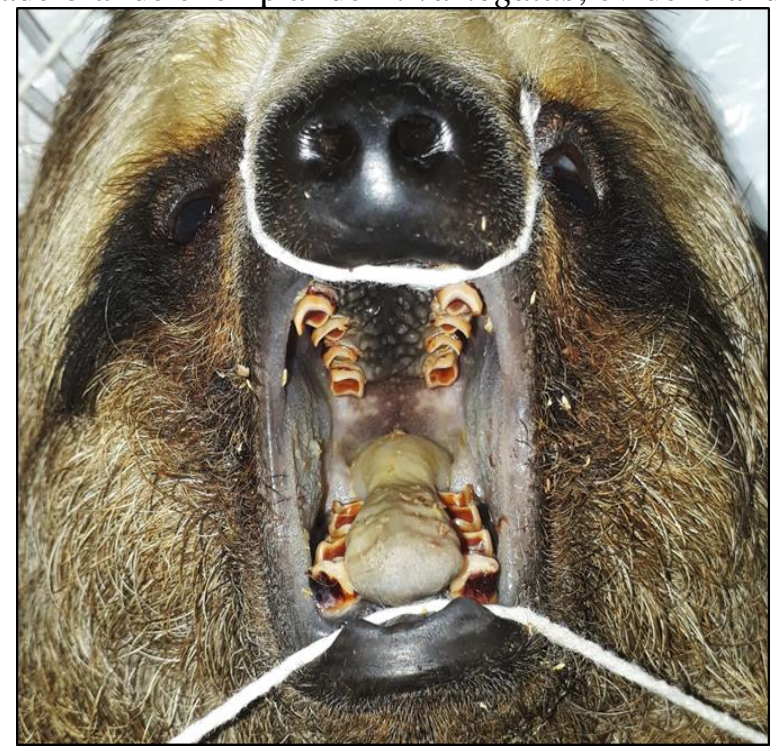

Fonte: Arquivo pessoal. 
Figura 2 - Ilustração de vista bucal e oclusão dentária da Preguiça-comum.

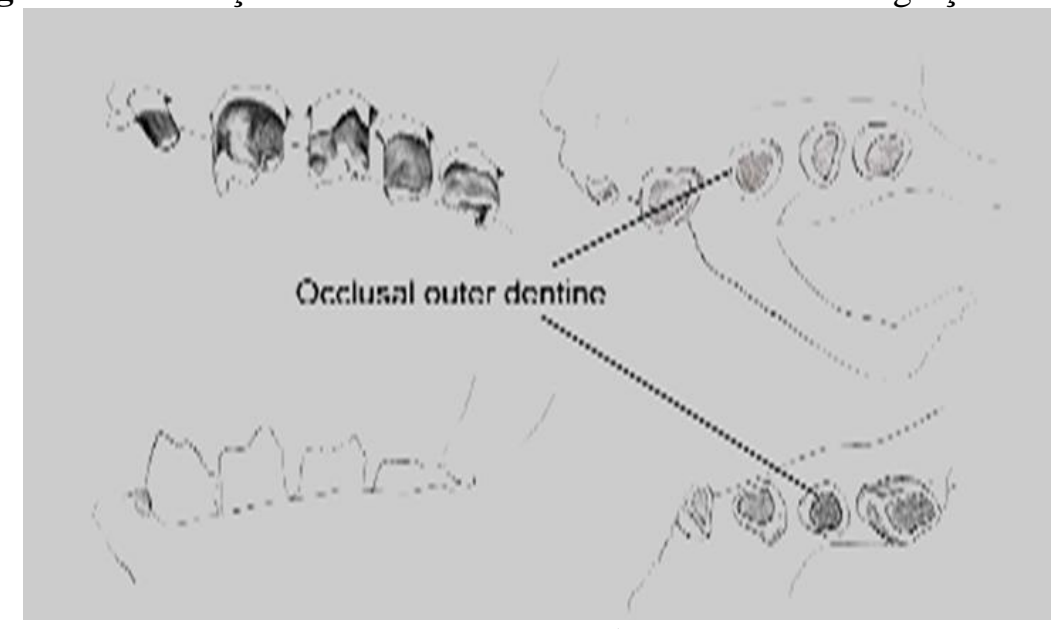

Fonte: Haupt et al., 2013.

O entendimento de padrões normais é essencial para reconhecer alterações patológicas, direcionando para o diagnóstico mais preciso em animais silvestres (ALVES, 2016). Por meio da radiografia (Figura 3) do crânio da espécie é possível evidenciar sua dentição, e identificar possíveis anormalidades. No osso maxilar nota-se a presença de cinco alvéolos dentários em cada hemiarcada. Já no osso mandibular, em sua borda alveolar, encontramos quatro molares (FREITAS, 2018).

Figura 3 - Radiografia de crânio de B. variegatus, com projeção lateral, sendo possível a visualização dos dentes.

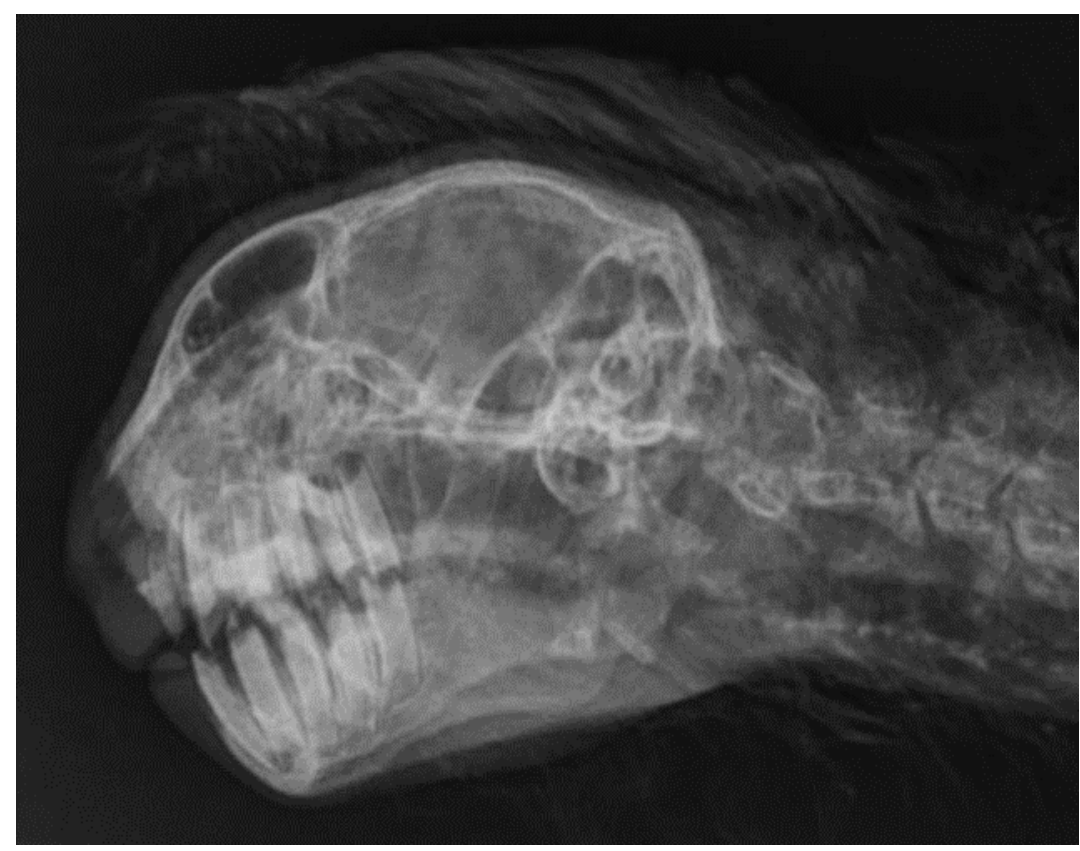

Fonte: Freitas, 2018. 


\section{Microscopia}

Albuquerque et al. (2016), realizou o exame microscópico dos dentes de exemplares de preguiça-comum através do desgaste e descalcificação. A técnica de desgaste consiste em polir os dentes com auxílio de uma lixa d'água, até atingir $2 / 3$ de sua espessura original, sendo observado o aspecto translúcido e, após esse procedimento, montar as lâminas para visualização em microscópio óptico (AZARIAS, 2005). Já na técnica de descalcificação, as amostras de dentes são colocadas em uma solução de ácido fórmico e citrato de sódio, que passa a ser renovada duas vezes por semana, por cerca de quatro meses, até que os dentes atinjam uma consistência gelatinosa. Completando a descalcificação, o material é processado histologicamente, e desidratado em etanol, sendo incluídos em parafina e submetidos a cortes de $5 \mu \mathrm{m}$. As lâminas são coradas com hematoxilina-eosina e observadas em microscópio ótico (AZARIAS, 2005).

É possível notar a falta de esmalte dos dentes e suas camadas, sendo uma camada espessa de cemento (Figura 4), camada de dentina interna e externa (Figura 5), e polpa.

Figura 4 - Visualização microscópica da camada espessa de cemento a) corte longitudinal b) corte transversal. 400x.
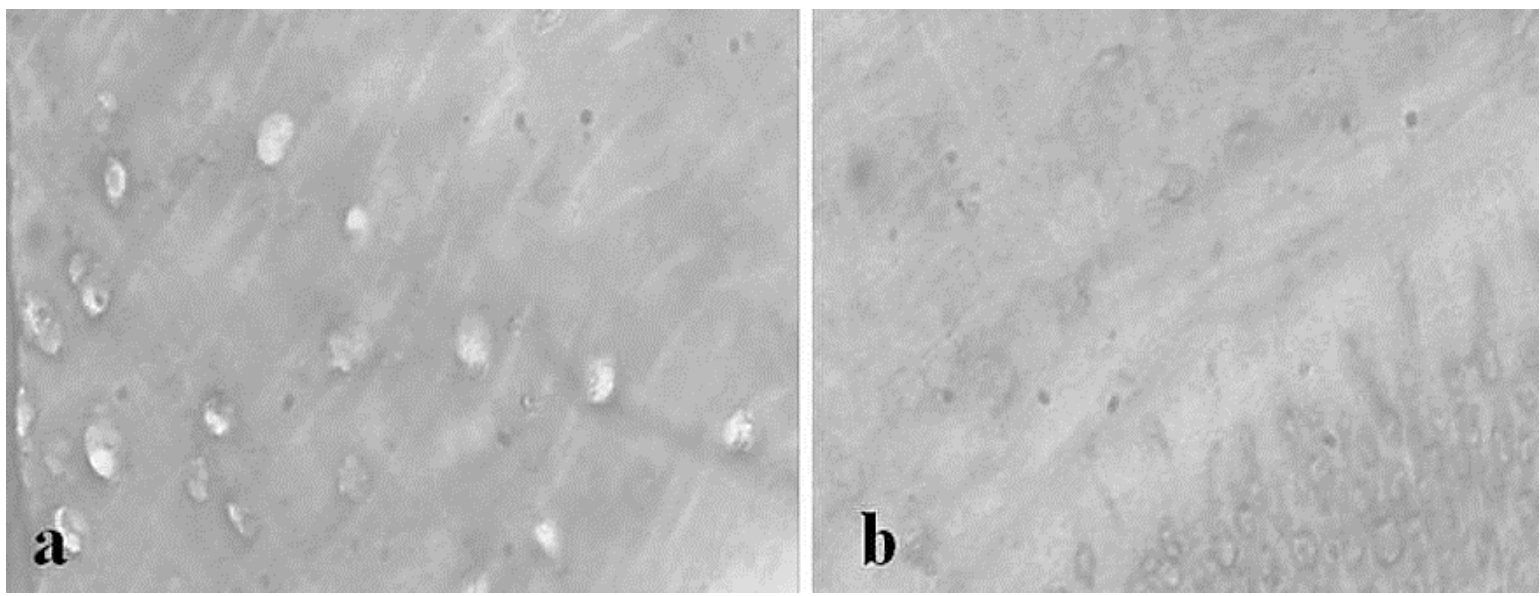

Fonte: Albuquerque et al., 2016. 
Figura 5 - Visualização microscópica da dentina interna (DI) e externa (DE), obtida através da técnica de desgaste. $400 \mathrm{x}$.

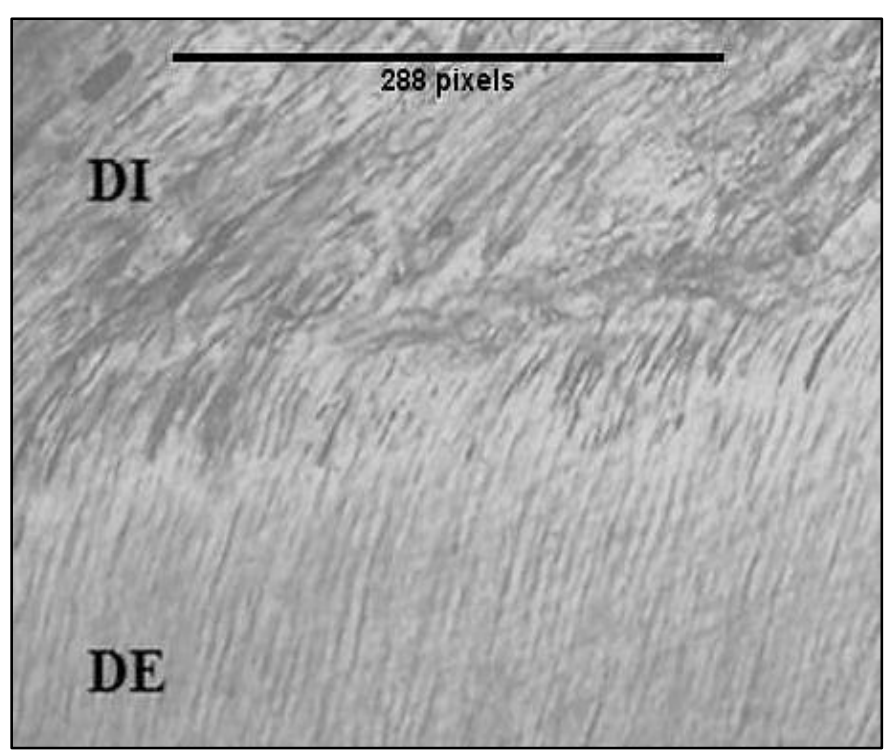

Fonte: Albuquerque et al., 2016.

A dentina exterior pode estar associada com a função de reparo, devido ao fato que a ausência de esmalte pode resultar em um desgaste mais rápido dos dentes e a polpa se assemelha à do ser humano (AZARIAS, 2005; ALBUQUERQUE et al., 2016).

Há presença de um ligamento periodontal espesso e bem vascularizado entre o osso e o dente, o que permite a visualização de alvéolos (Figura 6).

Figura 6 - Visualização microscópica de cemento (C), ligamento periodontal (Li) e osso alveolar (OA). 400x.

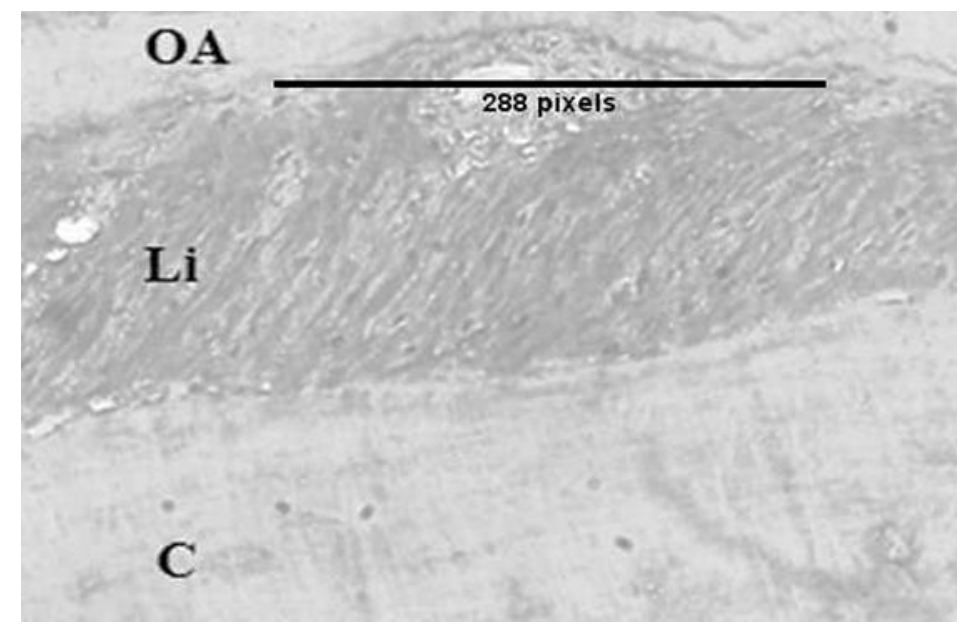

Fonte: Albuquerque et al., 2016. 
Outra técnica de avaliação microscópica, descrita em literatura por Haupt et al. (2012), em Xenarthra, consiste em avaliar os micros desgastes dentários (Figura 7), relacionando com a dieta do animal, e, desta forma, identificar, de acordo com a textura encontrada, o tipo de alimentação das espécies.

Figura 7 - Simulação 3D de digitalizações de textura superficial dos dentes de Bradypus variegatus.

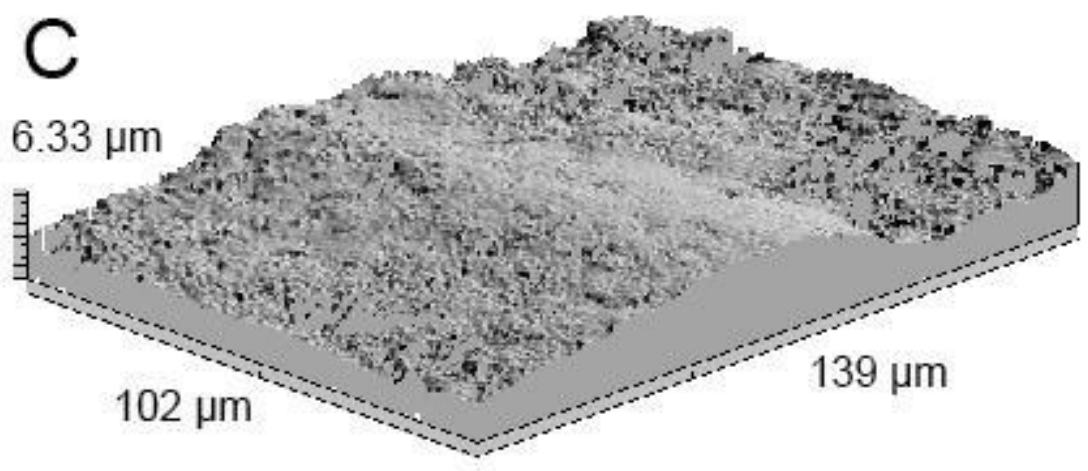

Fonte: Haupt et al., 2012.

\section{$\underline{\text { Anomalias }}$}

As variações dentárias ou anomalias, entre os mamíferos, podem ser divididas em duas principais categorias: de adição (hiperdontia) e deleção (anodontia), conforme descrito por Miles e Grigson (1990). McAfee (2015) analisou a dentição de 473 exemplares de Bradypus variegatus pertencentes a museus dos Estados Unidos, originárias de diversos locais da América Central e do Sul, buscando possíveis variações nessa espécie. Do total de animais analisados, 12 apresentaram anodontia e nove hiperdontia, além desses animais, 21 apresentaram as duas alterações simultaneamente.

Os casos de anodontia (Figura 8) foram mais frequentes em preguiça-comum. Essa condição é definida pela ausência completa do dente e fechamento do alvéolo, e, na maioria dos espécimes avaliados, não havia indicações ou cicatrizes que sugerissem que o dente estivesse presente anteriormente, podendo ser uma falha de desenvolvimento embriológico do dente (McAFEE, 2014). 
Figura 8 - Anodontias em maxilar registradas em 4 exemplares de Bradypus variegatus.

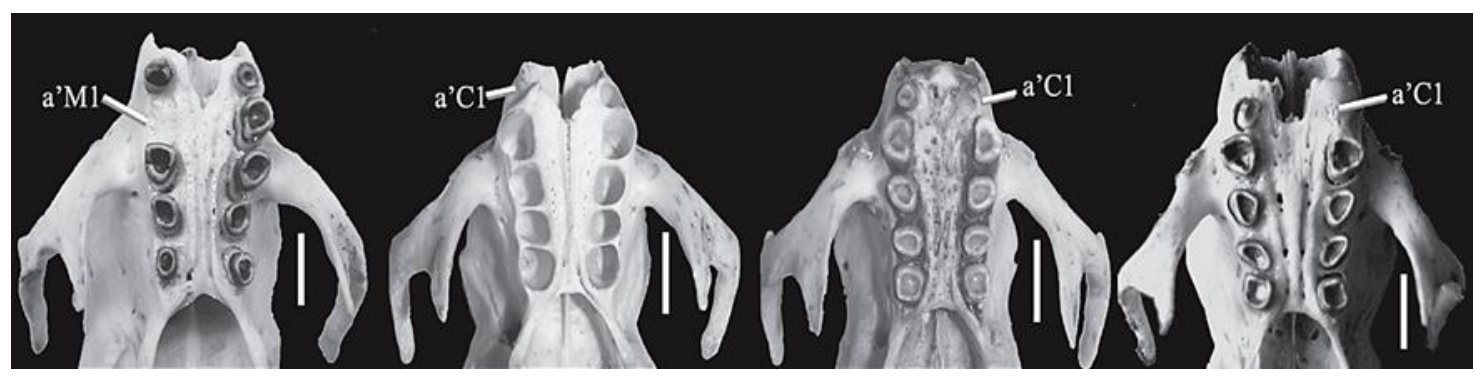

Fonte: McAfee, 2014.

Na hiperdontia (Figura 9), caracterizada pelo aumento no número de dentes, que são classificados como suplementares, os casos foram mais frequentes em outras espécies de preguiças (McAFEE, 2014).

Figura 9- Hiperdontia em maxilar de exemplar de B. variegatus.

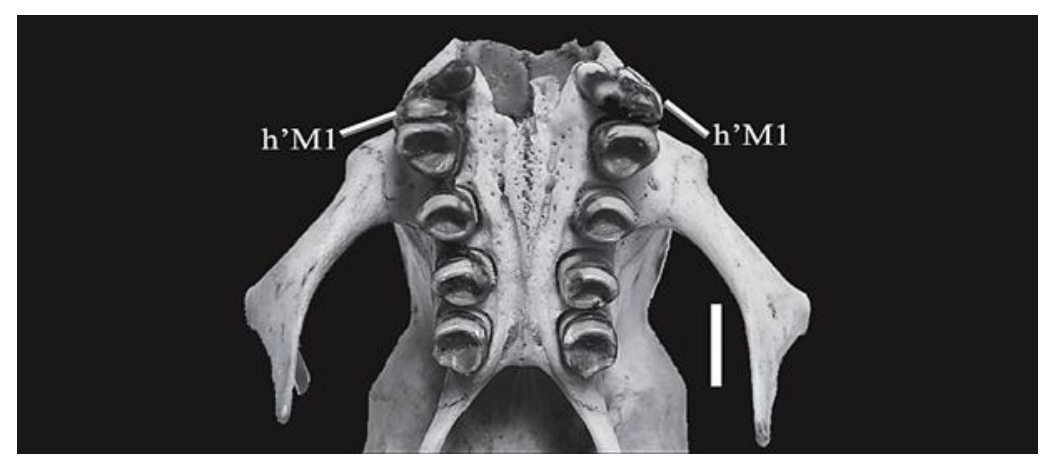

Fonte: McAfee, 2014.

\section{CONCLUSÃO}

A preguiça-comum é um animal que ainda exige muitos estudos para que se alcance maior conhecimento dos seus aspectos biológicos e fisiológicos. A evolução nesse aspecto é crescente, e para que continue assim, é necessário expandir o campo de estudo, não apenas visando a espécie, mas também seu habitat.

As ameaças são constantes e, com a crescente aproximação das cidades às florestas, surgem novos problemas, como atropelamentos, choques elétricos, ataque de animais domésticos e a retirada ilegal desses espécimes, de sua mata de origem, contribuindo para que os animais cheguem aos centros de reabilitação cada vez mais debilitados, exigindo que os profissionais da área se aperfeiçoem e sigam em constante progresso em prol do bem-estar dos animais silvestres.

O caminho para compreender as particularidades do aparelho digestório da preguiça-comum inicia-se a partir de seus dentes, especializados na maceração de 
alimentos vegetais e, com o avanço da tecnologia e surgimento de novas técnicas, é possível ir muito além nas descobertas relacionadas a este assunto, melhorando, dessa forma, o manejo do bicho-preguiça sob cuidados humanos, fazendo com que as chances de sucesso na reabilitação sejam cada vez maiores.

\section{REFERÊNCIAS}

ALBUQUERQUE, P.V.; SANTOS, F.R.; GALVÃO, A.; JÚNIOR, F.A.; ROSAS, E.P.; D'EMERY, M.B., FREITAS, M.F. Morphological analysis of teeth in Bradypus variegatus Schinz, 1825 (Mammalia, Bradypodidae). Biotemas, v. 29 n. 3, 2016.

ALVES, L. S. Descrição anatômica das estruturas osteoarticulares do esqueleto Axial do tatu-galinha (Dasypys novemcinctus Linnaeus, 1758) por meio de radiografia e tomografia computadorizada. Dissertação de Mestrado, Faculdade de Medicina Veterinária e Zootecnia da UNESP - Campus de Botucatu, 72p, 2016.

AZARIAS, R. E. G. R. Morfologia dos dentes do bicho-preguiça de coleira (Bradypus torquatus), Illiger, 1811. São Paulo, 2005.

BEZERRA, B. M.; SOUTO, A. S.; HALSEY, L.; SCHIEL, N. Observation of brownthroated three-toed sloths: mating behaviour and the simultaneous nurturing of two young. Journal of Ethology, v. 26, p. 175-178, 2008.

CASSANO, C. R. Ecologia e Conservação da Preguiça-de-Coleira (Bradypus torquatus Illiger, 1811) no Sul da Bahia. 2006. Dissertação de Mestrado - (Programa de Pós-Graduação em Zoologia). Universidade Estadual de Santa Cruz, Ilhéus, Bahia.

CHIARELlO, A. G. Sloth Ecology: An Overview of Field Studies. In: The Biology of the Xenarthra. VISCAÍNO, S. F.; LOUGHRY, W. J. Flórida, USA, 2008.

DINIZ, L. S. M.; OLIVEIRA, P. M. A. Clinical problems of sloths (Bradypus sp. and Choloepus sp) in captivity. J. Zoo. Wildl. Med., v. 30, p. 7680, 1999.

FREITAS, Kelvis de Brito. Estudo das variações anátomo-radiográficas do esqueleto do Bicho-Preguiça-de-Garganta-Marrom (Bradypus variegatus, SCHINZ, 1825) AREIA 2018. 2018.

GOFFART, M. Function and Form in the Sloth. Pergamon Press, Oxford, New York, Toronto, Sydney, Braunschweig, 1971.

HAUPT, R.J.; DESANTIS, L.R.G.; GREEN, J.L.; UNGAR, P.S. Dental microwear texture as a proxy for diet in xenarthrans. Journal of Mammalogy, 2013.

HAYSSEN, V. Bradypus variegatus (Pilosa: Bradypodidae). Mammalian Species 42: 19-32, 2010.

ITIS GLOBAL - The Integrated Taxonomic Information System. Detalhes das espécies: Bradypus variegatus Schinz, 1825. Acesso em 15/05/2019 
<http://www.catalogueoflife.org/col/details/species/id/d9bc399e42c7d82630f8016edaa2 $44 \mathrm{~cd}>$.

IUCN/SSC Anteater - Sloth and Armadillo Specialist Group. The 2010 Sloth Red List Assessment. Edentata (2010). Acesso em 18/05/2019

$<$ http://www.xenarthrans.org/species/details/category/2/specie/42/Preguiccedilacomum +ou+preguiccediladeoacuteculos $>$.

LOUREIRO, M.C.; MONTEIRO, A. R. Famílias de mamíferos brasileiros. Viçosa: Editora UFV, 1993. 105p.

MACHADO, A. B. M.; DRUMMOND, G. M.; PAGLIA, A.; Livro vermelho da fauna brasileira ameaçada de extinção. Brasília: Fundação Biodiversitas; 2008.

MCAFEE R. K. Dental anomalies within extant members of the mammalian Order Pilosa. Acta Zool. v. 96, p. 301-311, 2014.

MEDRI, I. M.; MOURÃO, G. M.; RODRIGUES, F. H. Ordem Pilosa In: DOS REIS, N. R.; PERACCHI, A. L.; PEDRO, N. A.; LIMA, I. P. Mamíferos do Brasil. 2a ed. Londrina: Nelio R. dos Reis, p. 91-95, 2011.

MILES, A. E. W.; GRIGSON, C.; COLYER, F. Colyer's variations and diseases of the teeth of animals. Cambridge, Cambridge University Press. 1990.

MIRANDA, F. R.; MESSIAS COSTA, A. Xenarthras (tamanduás, tatu e preguiça). In: CUBAS, Z. S.; SILVA, J. C. R.; CATÃO DIAS, J. L. Tratado de animais selvagens Medicina veterinária. São Paulo: Roca,. cap. 26, 2007.

MORAES-BARROS N.; CHIARELLO A.; PLESE T. Bradypus variegatus. The IUCN Red List of Threatened Species. Version 2015.2. www.iucnredlist.org. 2014.

MORAES-BARROS N.; SILVA J.A.; MIYAKI C.Y.; MORGANTE J.S. Comparative phylogeography of the Atlantic Forest endemic sloth (Bradypus torquatus) and the widespread three-toed sloth (Bradypus variegatus) (Bradypodidae, Xenarthra).

Genetica, v.126, n.1-2, p.189-98, 2006.

MOREIRA D.; LEITE G.R.; SIQUEIRA M.F.; MENDES S.L. The distributional ecology of the maned sloth: environmental influences on its distribution and gaps in knowledge; Plos one, v.9, n.10, p.110, 2014.

OLIVEIRA, V. S. Preservação Bicho Preguiça. CEPLAC/CEPEC. Itabuna, 2005. Disponível em: ceplac.gov.br/preguiça

PLESE, T.; CHIARELLO, A. Choloepus hoffmanni. In: IUCN. IUCN Red List of Threatened Species. 2 p., 2012.

REZENDE L.C.; GALDOS-RIVEROS A.C.; MIGLINO M.A. et al. Aspectos da biologia reprodutiva em preguiça e tamanduá: uma revisão. Revista Brasileira de Reprodução Animal, v.37, n. 4, p. 354-359, 2013. 
SILVA S.M.; SUMMA J.L.; SUMMA M.E.L.; GERALDI V.C.; BELLUCI M.; KLEFASZ A.; MORGANTE J.S.; MORAES-BARROS N. Contribution of wildlife governmental centers to conservation and biological study of sloths Bradypus variegatus. Natureza \& Conservação - Brazilian Journal of Nature Conservation, v.12, p.79-85, 2014.

SUPERINA, M.; MIRANDA, F.; PLESE, T. Maintenance of Xenarthra in captivity. In: VIZCAINO, S. F.; LOUGHRY, W. J. (eds.) The biology of Xenarthra. Florida: University Press of Florida, p. 232-243, 2008.

URBANI, B., BOSQUE, C. Feeding ecology and postural behaviour of the threetoed sloth (Bradypus variegatus flaccidus) in northern Venezuela. Mammalian Biology, 2007.

WETZEL, R. M. The Identification and Distribution of Recent Xenarthra (= Edentata). In: The Biology of the Xenarthra. VISCAÍNO, S. F.; LOUGHRY, W. J. Flórida, USA, 1985.

\section{Recebido em: 08/03/2022}

Aprovado em: 05/03/2022 